\title{
Finite generation of iterated wreath products
}

\author{
Ievgen V. Bondarenko
}

March 28, 2022

\begin{abstract}
Let $\left(G_{n}, X_{n}\right)$ be a sequence of finite transitive permutation groups with uniformly bounded number of generators. We prove that the infinitely iterated permutational wreath product ... $₹ G_{2} \curvearrowright G_{1}$ is topologically finitely generated if and only if the profinite abelian group $\prod_{n>1} G_{n} / G_{n}^{\prime}$ is topologically finitely generated. As a corollary, for a finite transitive group $G$ the minimal number of generators of the wreath power $G \imath \ldots \imath G<G$ ( $n$ times) is bounded if $G$ is perfect, and grows linearly if $G$ is non-perfect. As a by-product we construct a finitely generated branch group, which has maximal subgroups of infinite index, answering [2, Question 14].
\end{abstract}

Keywords: iterated wreath product, profinite group, inverse limit, branch group Mathematics Subject Classification 2000: 20F05, 20E22, 20E18, 20E08

\section{Introduction}

Let $(G, X)$ and $(H, Y)$ be permutation groups (all actions in the paper are faithful). The permutational wreath product $H \prec G$ is the semidirect product $H^{|X|} \rtimes G$ with a natural action on $X \times Y$, where $G$ acts on $H^{|X|}$ by permuting the copies of $H$. Given a sequence of finite permutation groups $\left(G_{n}, X_{n}\right)$ the inverse limit of iterated wreath products

$$
W=\lim _{\longleftarrow}\left(G_{n} \prec \ldots \succ G_{2} \prec G_{1}\right)
$$

is a profinite group called the infinitely iterated wreath product . . ₹ $G_{2} \prec G_{1}$. The goal of this note is to prove the following theorem.

Theorem 1. Let $\left(G_{n}, X_{n}\right)$ be a sequence of finite transitive permutation groups with uniformly bounded number of generators. Then the profinite group ... ? $G_{2} 2 G_{1}$ is finitely generated if and only if the profinite abelian group $\prod_{n \geq 1} G_{n} / G_{n}^{\prime}$ is finitely generated.

Since the group $\prod_{n>1} G_{n} / G_{n}^{\prime}$ is an epimorphic image of the group ... $2 G_{2} \prec G_{1}$, in one direction the statement is obvious. For the converse we construct a finitely generated dense subgroup. The construction is based on the notions of directed automorphisms and branch groups introduced by R. Grigorchuk [5], and it is basically the same construction used by 
P. Neumann [8], L. Bartholdi [1], D. Segal [11] and others to construct certain groups with interesting properties.

M. Bhattacharjee [3] showed that the infinitely iterated wreath products of alternating groups of degree $\geq 5$ can be generated by two elements even with positive probability. This was generalized to wreath products of non-abelian simple groups with transitive actions by M. Quick [9, 10]. D. Segal [1] showed that the infinitely iterated wreath products of perfect groups with certain conditions on the actions are finitely generated. The iterated wreath products of cyclic groups of pairwise coprime orders are two-generated by result of A. Woryna [14.

The profinite group $W$ is finitely generated if and only if the sequence $d\left(G_{n} \prec \ldots \curlywedge G_{2} \prec G_{1}\right)$ is bounded, where $d(G)$ is the minimal number of generators of the group $G$. The behavior of generating sequence $d\left(G^{n}\right)$ for the direct product $G^{n}$ of a finite group $G$ was described in a series of papers by J. Wiegold (see [12, 13] and references therein): the sequence $d\left(G^{n}\right)$ is roughly logarithmic if $G$ is perfect, and is linear otherwise. The generating sequence $d_{n}^{w r}(G)=d(G \succ \ldots \imath G \prec G)$ for the wreath power of a finite transitive group $G$ happens to be even more simple. As a corollary of Theorem 10 we get that $d_{n}^{w r}(G)$ is bounded if $G$ is perfect, and grows linearly otherwise.

The wreath powers of finite groups are related to self-similar groups of finite type introduced by R. Grigorchuk [6, Section 7] to describe profinite completion of certain branch groups. Every such group is given by a finite pattern, and there is an open question [6, Problem 7.3]: which patterns define finitely generated groups. Every self-similar group of finite type given by pattern of size 1 is isomorphic to the infinite wreath power ... $\{$ ₹ $G$. Hence it will be finitely generated if and only if the group $G$ is perfect. Moreover, in this case the finitely generated dense subgroup constructed in the proof of Theorem 1 is a branch group, which has maximal subgroups of infinite index. This answers Question 14 in [2, p. 1107].

Acknowledgments. I would like to thank Rostyslav Kravchenko and Dmytro Savchuk for fruitful discussions.

\section{Automorphisms of spherically homogeneous rooted tree}

Let $\mathcal{T}$ be the spherically homogeneous rooted tree given by the alphabets $X_{i}$, where the $n$-th level is $\mathcal{T}_{n}=X_{1} \times \ldots \times X_{n}$ (here $\mathcal{T}_{0}$ consists of the root $\emptyset$ ), and each vertex $v \in \mathcal{T}_{n}$ is connected with $v x \in \mathcal{T}_{n+1}$ for every $x \in X_{n+1}$. Denote by $\mathcal{T}^{[n]}$ the finite truncated rooted tree consisting of levels from 0 to $n$. For a vertex $v \in \mathcal{T}$ denote by $\mathcal{T}_{v}$ the rooted tree hanging "below" the vertex $v$, which consists of vertices $u$ such that the geodesic connecting $u$ with the root $\emptyset$ passes through $v$. The set $v X_{n}$ is the first level of tree $\mathcal{T}_{v}$ for $v \in \mathcal{T}_{n-1}$.

Every automorphism $g \in$ Aut $\mathcal{T}$ induces a map $v X_{n} \rightarrow g(v) X_{n}$ for every vertex $v \in$ $\mathcal{T}_{n-1}$. Forgetting the prefixes $v$ and $g(v)$ we get a permutation on the set $X_{n}$, which is called the vertex permutation of $g$ at $v$ and denoted $g \circledast v \in \operatorname{Sym}\left(X_{n}\right)$. Every automorphism can be given by its vertex permutations at each vertex. 
The automorphism group of the tree $\mathcal{T}^{[n]}$ is the iterated permutational wreath product

$$
\text { Aut } \mathcal{T}^{[n]}=\operatorname{Sym}\left(X_{n}\right) \text {.. } 2 \operatorname{Sym}\left(X_{1}\right),
$$

and the group Aut $\mathcal{T}$ is the inverse limit of these groups, which is also the infinite permu-

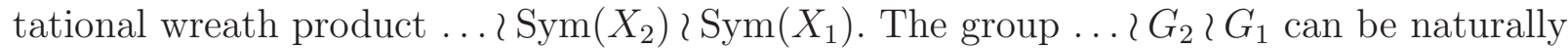
considered as a subgroup of Aut $\mathcal{T}$. We also identify the group $G_{1}$ with the subgroup of Aut $\mathcal{T}$, which consists of automorphisms whose vertex permutations at the root form the group $G_{1}$ and are trivial at the other vertices.

Let $G$ be a subgroup of Aut $\mathcal{T}$. The vertex stabilizer $\operatorname{St}_{G}(v)$ of a vertex $v \in \mathcal{T}$ is the subgroup of all $g \in G$ such that $g(v)=v$. The $n$-th level stabilizer $\operatorname{St}_{G}(n)$ is the subgroup of all $g \in G$ such that $g(v)=v$ for every $v \in \mathcal{T}_{n}$. The rigid vertex stabilizer $\operatorname{RiSt}_{G}(v)$ of a vertex $v \in \mathcal{T}$ is the subgroup of all $g \in G$ such that the vertex permutations of $g$ at vertices outside the subtree $\mathcal{T}_{v}$ are trivial. The rigid level stabilizer $\operatorname{RiSt}_{G}(n)$ is the subgroup generated by $\operatorname{RiSt}_{G}(v)$ for $v \in \mathcal{T}_{n}$. The group $G$ is called branch, if it acts transitively on the levels $\mathcal{T}_{n}$ and every rigid level stabilizer $\operatorname{RiSt}_{G}(n)$ is of finite index in $G$.

We say that the rigid stabilizer $\operatorname{RiSt}_{G}(v)$ for $v \in \mathcal{T}_{n-1}$ contains a subgroup $H \in \operatorname{Sym}\left(X_{n}\right)$ as a rooted subgroup, if $\operatorname{RiSt}_{G}(v)$ contains a subgroup whose vertex permutations at $v$ form the group $H$ and all the other vertex permutations are trivial.

\section{Proof of Theorem $\square$ and Corollaries}

Proof. (of Theorem (1) The trivial groups $G_{n}$ can be omitted and we can assume $\left|X_{n}\right| \geq 2$ for all $n$. Also we can assume that every group $G_{n}$ is non-abelian, otherwise we can pass to

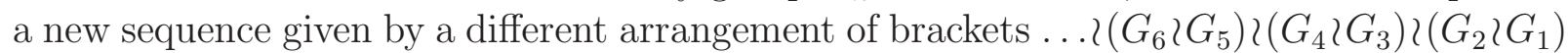
for which it is true (the permutational wreath product is associative). By the same reason (passing to the same arrangement of brackets with already non-abelian groups) we can assume that for every action $\left(G_{n}^{\prime}, X_{n}\right)$ there are points in the same orbit with different stabilizers. Fix letters $x_{n}, y_{n} \in X_{n}$ and permutations $\tau_{n}, \pi_{n} \in G_{n}^{\prime}$ such that $\tau_{n}\left(x_{n}\right)=y_{n}$, $\pi_{n}\left(x_{n}\right)=x_{n}$, and $\pi_{n}\left(y_{n}\right) \neq y_{n}$.

Consider the decomposition of the groups $G_{n} / G_{n}^{\prime}$ is the direct sum of cyclic groups of prime-power order. Since the group $\prod_{n>1} G_{n} / G_{n}^{\prime}$ is finitely generated, there is an absolute bound on the number of cyclic $p$-groups in these decomposition for any particular prime number $p$. Hence there is a finite generating set $a_{1}=\left(a_{1}^{(n)}\right)_{n}, \ldots, a_{e}=\left(a_{e}^{(n)}\right)_{n}$ such that for every fixed $i$ two elements $a_{i}^{(j)}$ and $a_{i}^{\left(j^{\prime}\right)}$ have coprime orders for every $j, j^{\prime}$. Let $g_{i}^{(n)} \in G_{n}$ be a preimage of $a_{i}^{(n)}$ under the canonical projection $G_{n} \rightarrow G_{n} / G_{n}^{\prime}$. Then $G_{n}=\left\langle g_{1}^{(n)}, \ldots, g_{e}^{(n)}, G_{n}^{\prime}\right\rangle$ for every $n$. Since there is a uniform bound on the number of generators of $G_{n}$, we can complete the elements $g_{1}^{(n)}, \ldots, g_{e}^{(n)}$ to a generating set of $G_{n}$ by some elements $g_{e+1}^{(n)}, \ldots, g_{m}^{(n)}$, i.e. $G_{n}=\left\langle g_{1}^{(n)}, \ldots, g_{m}^{(n)}\right\rangle$ for every $n$ with fixed $m$. Define the automorphisms $g_{1}, \ldots, g_{m}$ of the tree $\mathcal{T}$ by their vertex permutations

$$
g_{i} @ v= \begin{cases}g_{i}^{(k+1)}, & \text { if } v=y_{1} y_{2} \ldots y_{k-1} x_{k} ; \\ 1, & \text { otherwise. }\end{cases}
$$


Consider the group $G=\left\langle g_{1}^{(1)}, \ldots, g_{m}^{(1)}, g_{1}, \ldots, g_{m}\right\rangle$ and let us prove that $G$ is dense in the group $W$. Fix $n$ and let us show that the restriction of $G$ on the tree $\mathcal{T}^{[n]}$ coincides with the group $G_{n} \imath \ldots \curlywedge G_{2} \prec G_{1}$.

Since the group $G$ acts transitively on the set $X_{1}$ and the vertex stabilizer $\operatorname{St}_{G}\left(y_{1} \ldots y_{n-2} x_{n-1}\right)$ acts transitively on $y_{1} \ldots y_{n-2} x_{n-1} X_{n}$ for every $n$, inductively we get that the group $G$ acts transitively on the levels of $\mathcal{T}$.

Let us prove that the rigid vertex stabilizers $\operatorname{RiSt}_{G}\left(y_{1} \ldots y_{k-1} y_{k}\right)$ and $\operatorname{RiSt}_{G}\left(y_{1} \ldots y_{k-1} x_{k}\right)$ contain the group $G_{k+1}^{\prime}$ as a rooted subgroup. Since elements $g_{1}^{(1)}, \ldots, g_{m}^{(1)}$ generate the group $G_{1}$ as a rooted subgroup at the root of the tree, the statement holds at zero level. Assume that we have proved it for all levels $<k$. By inductive hypothesis there exist $\pi, \tau \in G$ such that $\pi \circledast y_{1} \ldots y_{k-1}=\pi_{k}, \tau \circledast y_{1} \ldots y_{k-1}=\tau_{k}$, and all the other vertex permutations are trivial. Then

$$
\begin{array}{r}
\pi^{-1} g_{i} \pi \circledast y_{1} \ldots y_{k-1} x_{k}=g_{i}^{(k+1)}, \quad \pi^{-1} g_{i} \pi \circledast y_{1} \ldots y_{s-1} x_{s}=1 \quad \text { for } s>k \\
\Rightarrow \quad\left[\pi^{-1} g_{i} \pi, g_{j}\right] \circledast y_{1} \ldots y_{l-1} x_{l}=\left[g_{i}^{(l+1)}, g_{j}^{(l+1)}\right] \quad \text { for } l \leq k
\end{array}
$$

and all the other vertex permutations of $\left[\pi^{-1} g_{i} \pi, g_{j}\right]$ are trivial for all $g_{i}, g_{j}$. By inductive hypothesis, we can multiply $\left[\pi^{-1} g_{i} \pi, g_{j}\right]$ on the appropriate elements from $\operatorname{RiSt}_{G}\left(y_{1} \ldots y_{l-1} x_{l}\right)$ for $l<k$ to remove all commutators at vertices $y_{1} \ldots y_{l-1} x_{l}$, and get elements from $\operatorname{RiSt}_{G}\left(y_{1} \ldots y_{k-1} x_{k}\right)$. Conjugating by generators $g_{1}, \ldots, g_{m}$ we get that $\operatorname{RiSt}_{G}\left(y_{1} \ldots y_{k-1} x_{k}\right)$ contains $G_{k+1}^{\prime}$ as a rooted subgroup. Conjugating by $\tau$ we get that $\operatorname{RiSt}_{G}\left(y_{1} \ldots y_{k-1} y_{k}\right)$ contains $G_{k+1}^{\prime}$ as a rooted subgroup.

The elements $a_{i}^{(1)}, \ldots, a_{i}^{(n)}$ have pairwise coprime orders (here $i \leq e$ ), and for a fixed $k \leq n$ we can choose a power $\alpha$ such that $\left(g_{i}\right)^{\alpha} \circledast y_{1} \ldots y_{k-2} x_{k-1}=g_{i}^{(k)} h_{i}$ for some $h_{i} \in G_{k}^{\prime}$, $\left(g_{i}\right)^{\alpha} \circledast y_{1} \ldots y_{l-2} x_{l-1} \in G_{l}^{\prime}$ for all $l \leq n, l \neq k$, and all the other vertex permutations at the vertices of $\mathcal{T}^{[n]}$ are trivial. Multiplying $\left(g_{i}\right)^{\alpha}$ on the corresponding elements from $\operatorname{RiSt}_{G}\left(y_{1} \ldots y_{l-2} x_{l-1}\right)$ we can remove the elements from commutants, and get element $f_{i} \in G$ such that $f_{i} \circledast y_{1} \ldots y_{k-2} x_{k-1}=g_{i}^{(k)}$ with all the other vertex permutations at the vertices of $\mathcal{T}^{[n]}$ being trivial. It follows that the group $G$ contains a subgroup $H$ such that $H \circledast y_{1} \ldots y_{k-2} x_{k-1}=G_{k}$ and the vertex permutations of every element of $H$ at the vertices of $\mathcal{T}^{[n]}$ and outside $\mathcal{T}_{y_{1} \ldots y_{k-2} x_{k-1}}$ are trivial. Since the action is transitive this holds for every vertex $v \in \mathcal{T}_{k-1}$. The result follows.

Remark 1. We got the bound on the number of generators. In some cases one can reduce the number of generators as it was done in [11] using Lemma 2 there.

The next corollary is a generalization of result in [14].

Corollary 2. For a sequence $A_{n}$ of finite abelian transitive groups with pairwise coprime orders and uniformly bounded number of generators the iterated wreath product . . $A_{2} 2 A_{1}$ is finitely generated.

The last corollary also follows from Theorem 2 in [7], which says that if $(G, X)$ is a finite transitive permutation group and $H$ is a finite solvable group, then

$$
d(H \succ G)=\max \left(d\left(H / H^{\prime} \prec G\right),\left[\frac{d(H)-2}{|X|}\right]+2\right) .
$$


Moreover, by Corollary 6 in [7], if $H$ is abelian of coprime order with $|G|$, then

$$
d(H \curlywedge G)=\max (d(G), d(H)+1) .
$$

Hence $d\left(A_{n} \nmid \ldots \imath A_{2} \prec A_{1}\right)=\max _{2 \leq i \leq n}\left(d\left(A_{1}\right), d\left(A_{i}\right)+1\right)$ under conditions of Corollary 2 .

The next example shows that we cannot remove assumption on the number of generators in Theorem 1 .

Example 1. Take a sequence of finite solvable transitive groups $\left(G_{n}, X_{n}\right)$ such that the groups $G_{n} / G_{n}^{\prime}$ are cyclic with pairwise coprime orders and $d\left(G_{n}\right)$ is greater than $n\left|X_{1}\right|\left|X_{2}\right| \cdots\left|X_{n-1}\right|$. Then the sequence $d\left(G_{n} \succ \ldots \succ G_{2} \prec G_{1}\right)$ is not bounded by (2), the group $W$ is not finitely generated, while the group $\prod_{n \geq 1} G_{n} / G_{n}^{\prime}$ is procyclic.

The next examples show that the uniform bound on the number of generators in not necessary for the group $W$ to be finitely generated.

Example 2. Take a sequence of finite solvable transitive groups $\left(G_{n}, X_{n}\right)$ such that every group $G_{n} / G_{n}^{\prime}$ is cyclic of order coprime with $G_{n-1} \imath \ldots \imath G_{2} \imath G_{1}$, and $\left|X_{1}\right|\left|X_{2}\right| \cdots\left|X_{n-1}\right|>$ $d\left(G_{n}\right) \rightarrow \infty$. Then the sequence $d\left(G_{n} \prec \ldots \curlywedge G_{2} \prec G_{1}\right)$ is bounded by (2) and (3), hence the group $W$ is finitely generated.

Example 3. Using result in [i] one can construct a sequence of finite perfect transitive groups $\left(G_{n}, X_{n}\right)$ for $n \geq 2$ with the following properties: there are generating sets $G_{n}=$ $\left\langle g_{1}^{(n)}, \ldots, g_{m_{n}}^{(n)}\right\rangle$ such that the elements $g_{i}^{(j)}$ have pairwise coprime orders for all $i, j$, and $\left|X_{n-1}\right|>d\left(G_{n}\right)=m_{n} \rightarrow \infty$ when $n \rightarrow \infty$. Take the cyclic group $G_{1}=\langle a\rangle$ of order $d\left(G_{2}\right)+1$ with the regular action $\left(G_{1}, X_{1}\right)$. Fix different letters $x_{n}^{(1)}, \ldots, x_{n}^{\left(m_{n}\right)}, y_{n} \in X_{n}$ for every $n$ and define the automorphism $b$ of the tree $\mathcal{T}$ by its vertex permutations

$$
b \circledast v= \begin{cases}g_{i}^{(n+1)}, & \text { if } v=y_{1} y_{2} \ldots y_{n-1} x_{n}^{(i)} ; \\ 1, & \text { otherwise. }\end{cases}
$$

Since the elements $g_{i}^{(j)}$ have coprime orders we can take a power of $b$ to get any $g_{i}^{(j)}$ rooted at vertex $y_{1} \ldots y_{j-2} x_{j-1}^{(i)}$ with all the other vertex permutations at vertices of $\mathcal{T}^{[n]}$ being trivial for every fixed $n$. Since the group $\langle a, b\rangle$ acts transitively on the levels of $\mathcal{T}$, conjugating we get the group $G_{j}$ as a rooted subgroup at every vertex of $(j-1)$-th level with all the other vertex permutations at vertices of $\mathcal{T}^{[n]}$ being trivial. Hence the group $\langle a, b\rangle$ is dense in $W$, while the abelianization of $W$ is the finite cyclic group $G_{1}$ and $d\left(G_{n}\right) \rightarrow \infty$.

Corollary 3. Let $H$ be a finite transitive group. The infinite wreath power ... $\mathrm{H} \imath \mathrm{H}$ is finitely generated if and only if the group $H$ is perfect.

Consider the previous corollary in more details. Let $H=\left\langle h_{1}, \ldots, h_{m}\right\rangle$ (we assume the group $H$ satisfies the first paragraph of the proof of Theorem [1, otherwise take $H<H$ ), then the dense group $G$ constructed in the proof of 
Theorem 1 is generated by $h_{1}, \ldots, h_{m}$ and $g_{1}, \ldots, g_{m}$, where every automorphism $g_{i}$ is defined recursively by

$$
g_{i}(v)= \begin{cases}x g_{i}(u), & v=x u \\ y h_{i}(u), & v=y u \\ v, & \text { otherwise }\end{cases}
$$

( $x=x_{1}$ and $y=y_{1}$ are from the theorem). Here $\left\langle g_{1}, \ldots, g_{m}\right\rangle \simeq H$, and hence the group $G$ is perfect, because it is generated by perfect subgroups. In the same way as in the proof of Theorem 1 1 we get that $\operatorname{St}_{G}(n)=\operatorname{RiSt}_{G}(n) \simeq G \times \ldots \times G$. In particular, the group $G$ is branch (actually regular branch over itself, see definition in [5]). It is also just-infinite by [5, Theorem 3], and satisfies the congruence subgroup property by [5, Proposition 2], i.e. every subgroup of finite index contains the stabilizer of some level. In particular, the group $W=\ldots$ \& $H$ < $H$ is not only the closure of the group $G$ in Aut $\mathcal{T}$, but also the profinite completion of $G$.

Consider the subgroup $F<G$, which consists of all automorphisms from $G$ that have only finitely many non-trivial vertex permutations (finitary automorphisms). Since $\operatorname{RiSt}_{G}(v)$ contains $H$ as a rooted subgroup for every vertex $v$, the group $F$ consists of all automorphisms of the tree $\mathcal{T}$, which have only finitely many non-trivial vertex permutations and all vertex permutations are from the group $H$. In particular, the subgroup $F$ is dense in the groups $W$ and $G$. Also $F$ is a proper subgroup of $G$ (here $g_{1}, \ldots, g_{m}$ are not in $F$ ). Since the group $G$ is finitely generated, the subgroup $F$ is contained in some maximal subgroup $M$, which is also dense in $G$ and hence of infinite index. Indeed, if $M$ had finite index then it would contain some level stabilizer $\operatorname{St}_{G}(n)$; but $M / \operatorname{St}_{G}(n)=G / \operatorname{St}_{G}(n)$ and we get $M=G$, which contradicts maximality of $M$. This answers Question 14 in [2, p. 1107] (see discussion in [6, Section 6]).

Corollary 4. Let $H$ be a finite transitive group and put $d_{n}^{w r}(H)=d(H \imath \ldots$ 々 $H$ 々 $H)$. The sequence $d_{n}^{w r}(H)$ is bounded if $H$ is perfect, and grows linearly otherwise.

Proof. As shown above, if $H$ is perfect then

$$
d(H) \leq d_{n}^{w r}(H) \leq 2 d(H \curlywedge H) .
$$

Let us show that if $H$ is non-perfect, then

$$
n d\left(H / H^{\prime}\right) \leq d_{n}^{w r}(H) \leq 2 d(H \succ H \curlywedge H \curlywedge H)+n d\left(H / H^{\prime}\right)
$$

(here $H \imath H \imath H \imath H$ is to make sure that the action of commutant satisfies the first paragraph of the proof of Theorem [1). The lower bound is clear. For the upper bound we proceed as in the proof of Theorem 1. If $H=\left\langle h_{1}, \ldots, h_{m}\right\rangle$ then we define the automorphisms $g_{1}, \ldots, g_{m}$ of the tree $\mathcal{T}^{[n]}$ by (1). For every generator $a$ of $H / H^{\prime}$ take its preimage $b$ in $H$ and construct $n-1$ automorphisms $b^{(1)}, \ldots, b^{(n-1)}$, where $b^{(i)} \circledast v=b$ if $v=y \ldots y x$ (here the length $=i$ ) and trivial otherwise. The group generated by all constructed elements is isomorphic to $H$ ?.. $2 H \imath H$. 


\section{References}

[1] L. Bartholdi. A Wilson group of non-uniformly exponential growth. C. R., Math., Acad. Sci. Paris, 336(7):549-554, 2003.

[2] L. Bartholdi, R. Grigorchuk, Z. Šuniḱ. Branch groups. In Handbook of algebra, vol. 3, pages 989-1112. North-Holland, Amsterdam, 2003.

[3] M. Bhattacharjee. The probability of generating certain profinite groups by two elements. Isr. J. Math., 86(1-3):311-329, 1994.

[4] C. David. Generating numbers for wreath products. Rend. Semin. Mat. Univ. Padova, 92:71-77, 1994.

[5] R. Grigorchuk. Branch groups. Math. Notes, 67(6):718-723, 2000.

[6] R. Grigorchuk. Solved and unsolved problems around one group. In Infinite groups: geometric, combinatorial and dynamical aspects. Progr. Math., vol. 248, pages 117-218. Birkhäuser, Basel, 2005.

[7] A. Lucchini. Generating wreath products and their augmentation ideals. Rend. Semin. Mat. Univ. Padova, 98:67-87, 1997.

[8] P. Neumann. Some questions of Edjvet and Pride about infinite groups. Ill. J. Math., 30:301-316, 1986.

[9] M. Quick. Probabilistic generation of wreath products of non-Abelian finite simple groups. Commun. Algebra, 32(12):4753-4768, 2004.

[10] M. Quick. Probabilistic generation of wreath products of non-Abelian finite simple groups. II. Int. J. Algebra Comput., 16(3):493-503, 2006.

[11] D. Segal. The finite images of finitely generated groups. Proc. Lond. Math. Soc., III. Ser., 82(3):597-613, 2001.

[12] J. Wiegold. Growth sequences of finite groups II. J. Austral. Math. Soc., 20(2):225229, 1975.

[13] J. Wiegold, J. Wilson. Growth sequences of finitely generated groups. Arch. Math., 30:337-343, 1978.

[14] A. Woryna. On generation of wreath products of cyclic groups by two state time varying Mealy automata. Int. J. Algebra Comput., 16(2):397-415, 2006. 